\title{
Verification of the scaling relation within MPDFT by analyzing PDFs for energy dissipation rates out of $4096^{3}$ DNS
}

\author{
N. Arimitsu \\ Graduate School of Environment and Information Sciences, Yokohama National University \\ Yokohama 240-8501, Japan \\ arimitsu@ynu.ac.jp \\ T. Arimitsu \\ Graduate School of Pure and Applied Sciences, University of Tsukuba \\ Ibaraki 305-8571, Japan
}

March 7, 2011

\begin{abstract}
The PDFs for energy dissipation rates, extracted by Kaneda and Ishihara from their $4096^{3}$ DNS for fully developed turbulence, are analyzed within the framework of multifractal probability density function theory (MPDFT) in a high accuracy. MPDFT is a statistical mechanical ensemble theory in order to analyze intermittent phenomena providing fat-tail probability density functions (PDFs). Recently, MPDFT has been improved by introducing a new scaling relation to be able to analyze intermittency through any series of PDFs with arbitrary magnification $\delta(>1)$. It had been fixed to a unique value $\delta=2$. Since the value of $\delta$ can be determined freely by observers, the choice of $\delta$ should not affect observables. The validity of the generalized MPDFT is verified successfully through the precise analyses of several series of PDFs with different values of $\delta$. In the course of the verification, it is revealed that the system of fully developed turbulence has much wider area representing scaling behaviors than the inertial range. With the help of MPDFT, it has become possible to separate the coherent turbulent motion from fluctuations. This may benefit the wavelet analysis of turbulence.
\end{abstract}

\section{Introduction}

In the keystone works $[1,2,3,4,5,6,7,8,9,10,11,12,13,14]$ providing the multifractal aspects for fully developed turbulence, only a few $[8,12,13,14,15,16,17,18,19,20,21]$ analyze the probability density functions (PDFs) extracted from experiments or numerical experiments. Most of the works deal with the scaling property of the system, e.g., comparison of the scaling exponents of velocity structure function. Among the researches analyzing PDFs, multifractal probability density function theory (MPDFT) [12, $13,15,16,18,19,21]$ provides the most precise analyses of the system including PDFs. MPDFT is a statistical mechanical ensemble theory for analyzing those phenomena providing fat-tail PDFs, which was constructed by the authors under the assumption, following Frisch and Parisi [2], that the singularities due to the scale invariance of the Navier-Stokes (N-S) equation for high Reynolds number distribute themselves multifractal way in real physical space. The degree of singularity for those quantities which are responsible for intermittent phenomena is specified by the singularity exponent $\alpha$ that is assumed to be equal to the parameter appeared in the scale transformation as an arbitrary parameter taking real values (see (5) and (6) below). Note that $\alpha$ is regarded as a stochastic variable, and is related definitely to the quantity representing intermittent behavior.

Within the approach of MPDFT, the p model [5], the log-normal model [22, 23, 24] and the A\&A model $[10,11,12,13,15,16,18,19,21]$ are categorized as models with increasing number of parameters in the probability density function for $\alpha$, which are determined self-consistently as functions of the intermittency exponent $\mu$. For the case of the p model, the distribution of $\alpha$ is given by the binomial distribution function with one parameter that is specified by the definition of $\mu$. For the log-normal 
model, the distribution of $\alpha$ is given by the Gaussian distribution function with two parameters which are determined by the condition for energy conservation and the definition of $\mu$. For the case of the A\&A model, the distribution of $\alpha$ is given by Tsallis-type distribution function with three parameters which are fixed by the condition of energy conservation, the definition of $\mu$ and the scaling relation. The extraction of an appropriate distribution for $\alpha$ may lead us to a resolution for the origin of intermittency in turbulence. MPDFT provides us with a systematic framework to make a connection between the distribution of $\alpha$ and the PDFs of observed quantities.

In order to extract the intermittent character of the fully developed turbulence, it is necessary to have information of hierarchical structure of the system. This is realized by producing a series of PDFs for responsible singular quantities with different lengths

$$
\ell_{n}=\ell_{0} \delta^{-n}, \quad \delta>1 \quad(n=0,1,2, \cdots)
$$

that characterize the sizes of eddies or the regions in which the physical quantities are coarse-grained. The value for $\delta$ is chosen freely by observers. Therefore, the choice of $\delta$ should not affect the theoretical estimation of the values for the fundamental quantities, i.e., observables characterizing the turbulent system under consideration. The A\&A model within the framework of MPDFT itself tells us that this requirement is satisfied if the scaling relation has the form ${ }^{1}$

$$
\frac{\ln 2}{(1-q) \ln \delta}=\frac{1}{\alpha_{-}}-\frac{1}{\alpha_{+}}
$$

Here, $q$ is the index associated with the Rényi entropy [27] or with the Havrda-Charvat [28] and Tsallis [29] (HCT) entropy; $\alpha_{ \pm}$are zeros of the multifractal spectrum (see below in sub-section 4.1). The multifractal spectrum is uniquely related to the PDF for $\alpha$ which is responsible for the tail part of PDFs for those quantities revealing intermittent behavior whose singularity exponents can have values $\alpha<1$. With the new scaling relation (2), observables have come to include the parameter $\delta$ only in the combination $(1-q) \ln \delta$. The difference in $\delta$ is absorbed in the entropy index $q$.

If we further introduce a new number $\tilde{n}$ by

$$
\tilde{n}=n \ln \delta
$$

in addition to the scaling relation (2), and assume that $\tilde{n}$ is independent of $\delta$, the PDF of $\alpha$ also becomes independent of $\delta$. Note that, with the new number $\tilde{n}, \ell_{n}$ introduced in (1) reduces to $\ell_{n}=\ell_{0} \mathrm{e}^{-\tilde{n}}$, and that $\tilde{n}$ may provide us with a common number of steps appropriate for the interpretation of turbulence within the cascade model.

In this paper, we analyze, in terms of MPDFT, the PDFs of energy dissipation rates, extracted by Kaneda and Ishihara from their $4096^{3}$ DNS for fully developed turbulence [30], which are given in the series with $\delta=2^{1 / 4}$, and check the validity of the above assumption. In section 2 , we introduce an exponent representing the degree of singularity for observables responsible to intermittent character of turbulence. The singularity originates from the invariance of the N-S equation under a scale transformation. The PDF for the singular exponents is introduced in section 3 in connection with the multifractal spectrum. Other materials necessary for multifractal description, such as the mass exponent, the generalized dimension and so on, are introduced. Section 4 provides the self-contained introduction of the framework of MPDFT with the new scaling relation, and the construction of the PDFs within A\&A model whose variable range extends both positive and negative regions. In section 5, we analyze in a high precision the observed PDFs for energy dissipation rates by the theoretical PDF within A\&A model of MPDFT, and verify the proposed assumption through the analysis. Summary and prospects are devoted in section 6 .

\footnotetext{
${ }^{1}$ Since almost all the PDFs that had been provided previously were for the case where $\delta=2$, it has been possible to analyze PDFs with the scaling relation $1 /(1-q)=1 / \alpha_{-}-1 / \alpha_{+}$proposed by Costa, Lyra, Plastino and Tsallis [25, 26] in the context of dynamical systems.
} 


\section{Scaling invariance and singularities}

We consider an incompressible fluid where the mass density $\rho=\rho(\vec{x}, t)$ of the fluid is constant in time and space. In this case, the N-S equation for the velocity field $\vec{u}=\vec{u}(\vec{x}, t)$ reduces to

$$
\frac{\partial \vec{u}}{\partial t}+(\vec{u} \cdot \vec{\nabla}) \vec{u}=-\vec{\nabla} p+\nu \nabla^{2} \vec{u}
$$

where we introduced $p=\hat{p} / \rho$, and the kinematic viscosity $\nu=\hat{\eta} / \rho$. Here, $\hat{p}=\hat{p}(\vec{x}, t)$ is the pressure of fluid, and $\hat{\eta}$ is the viscosity. The condition for incompressibility reduces to the equation representing that there is no divergence in velocity field, i.e., $\vec{\nabla} \cdot \vec{u}=0$. The N-S equation (4) is invariant under the scale transformation

$$
\vec{x} \rightarrow \vec{x}^{\prime}=\lambda \vec{x}, \quad \vec{u} \rightarrow \vec{u}^{\prime}=\lambda^{\alpha / 3} \vec{u}, \quad t \rightarrow t^{\prime}=\lambda^{1-\alpha / 3} t, \quad p \rightarrow p^{\prime}=\lambda^{2 \alpha / 3} p
$$

and

$$
\nu \rightarrow \nu^{\prime}=\lambda^{1+\alpha / 3} \nu
$$

with an arbitrary real number $\alpha$. By the way, in the region where the intermittency of turbulence is conspicuous, the effect of the dissipative term in the N-S equation is very small compared with those of other terms (especially, the drift term). Therefore, let us try to extract the phenomena which are invariant under the scale transformation (5) without (6). ${ }^{2}$ We utilize the invariance under this scale transformation in order to introduce at the zero-th order approximation the characteristics that the appearance of the velocity field for fully developed turbulence is invariant even if we change the scale (or the distance) of observation. However, we should keep in mind that the dissipative term can become effective depending on the region under consideration since the term breaking the invariance does exist, i.e., non-zero (see the discussions in the following).

Let us now find out what character the system has when it is invariant under the transformation (5). We start with the velocity difference (fluctuation) $\delta u_{n}=\left|u\left(\bullet+\ell_{n}\right)-u(\bullet)\right|$ of a component $u$ of the velocity field $\vec{u}$ between two points separated by the distance $\ell_{n}$, defined by (1), which provides us with the measure of diameters of eddies generated at the $n$th stage. The diameters of eddies being produced one after another become $\delta^{-1}$ times smaller at each generation of eddies from bigger ones. Turbulence is not fluctuating randomly but intermittently, i.e., in the intermittent rhythms. The energy dissipation rate $\varepsilon_{n}$ averaged in the regions with diameter $\ell_{n}$ is given by

$$
\varepsilon_{n} \sim \nu_{n}\left(\frac{\delta u_{n}}{\ell_{n}}\right)^{2}
$$

where $\nu_{n}$ is the kinematic viscosity associated with the scale under consideration. Note that the actual dissipation rates exist for $\ell_{n}<\eta$ where $\eta$ is the Kolmogorov scale.

The scale transformation (5) gives

$$
\frac{\delta u_{n}}{\delta u_{0}}=\left(\frac{\ell_{n}}{\ell_{0}}\right)^{\alpha / 3}, \quad \frac{\delta p_{n}}{\delta p_{0}}=\left(\frac{\ell_{n}}{\ell_{0}}\right)^{2 \alpha / 3} .
$$

The difference $\delta p_{n}=\left|p\left(\bullet+\ell_{n}\right)-p(\bullet)\right|$ is also an important observable. As for the energy dissipation rate $\varepsilon_{n}$, we need the scale transformation (6) giving

$$
\frac{\nu_{n}}{\nu_{0}}=\left(\frac{\ell_{n}}{\ell_{0}}\right)^{1+\alpha / 3}
$$

since the dissipative term is necessary for energy dissipation. Then, (7) gives us

$$
\frac{\varepsilon_{n}}{\epsilon}=\left(\frac{\ell_{n}}{\ell_{0}}\right)^{\alpha-1}
$$

where we put $\varepsilon_{0}=\epsilon$ which is the energy input rate into the turbulent system, and is asuumed to be constant.

The velocity derivative $u^{\prime}$ and the fluid particle acceleration $\vec{a}$ are described, ${ }^{3}$ respectively, by $\left|u^{\prime}\right|=$

\footnotetext{
${ }^{2}$ Strictly speaking, the N-S equation (4) is invariant under this transformation only for the case $\nu=0$, i.e., when the Reynolds number is infinite.

${ }^{3}$ The fluid particle acceleration $\vec{a}$ is given by $\vec{a}=\partial \vec{u} / \partial t+(\vec{u} \cdot \vec{\nabla}) \vec{u}$.
} 
$\lim _{n \rightarrow \infty} u_{n}^{\prime}$ and $|\overrightarrow{\mathrm{a}}|=\lim _{n \rightarrow \infty} \mathrm{a}_{n}$. Here, we introduced the velocity derivative $u_{n}^{\prime}$ and acceleration $\mathrm{a}_{n}$ corresponding to the characteristic length $\ell_{n}$ by $u_{n}^{\prime}=\delta u_{n} / \ell_{n}, \mathrm{a}_{n}=\delta p_{n} / \ell_{n}$, respectively. We see that the velocity derivative and the fluid particle acceleration, respectively, have singularities ${ }^{4}$ for $\alpha<3$ and $\alpha<1.5$, i.e., $\left|u^{\prime}\right| \propto \lim _{\ell_{n} \rightarrow 0} \ell_{n}^{(\alpha / 3)-1} \rightarrow \infty,|\overrightarrow{\mathrm{a}}| \propto \lim _{\ell_{n} \rightarrow 0} \ell_{n}^{(2 \alpha / 3)-1} \rightarrow \infty$. The energy dissipation rate also have singularities for $\alpha<1$, i.e., $\lim _{n \rightarrow \infty} \varepsilon_{n}=\lim _{n \rightarrow \infty} \ell_{n}^{\alpha-1} \rightarrow \infty$. The exponent $\alpha$ plays the role of an index representing the degree of singularities [2].

The scaling exponent $\zeta_{m}$ of the $m$ th order velocity structure function (the $m$ th moment of velocity fluctuations) defined through

$$
\left\langle\left(\frac{\delta u_{n}}{\delta u_{0}}\right)^{m}\right\rangle=\left(\frac{\ell_{n}}{\ell_{0}}\right)^{\zeta_{m}}
$$

is one of the quantities which characterizes turbulence. Here, $\langle\ldots\rangle$ indicates to take an appropriate time average, spatial average or ensemble average. Actually, the present main issue is to search for an appropriate PDF for the average.

Within the treatment of K41 [31, 32], as the energy dissipation rates $\varepsilon_{n}$ are assumed to be constant independent of $n$, we see from (10) that K41 is the case corresponding to $\alpha=1$, and that the scaling exponents for K41 are given by $\zeta_{m}=m / 3$. If we look at this way, the arbitrariness of $\alpha$, appeared in the scale transformation (5), indicates that $\varepsilon_{n}$ can be viewed as a stochastic variable, i.e., one can introduce fluctuations in $\varepsilon_{n}$. It means that there is a possibility to give an answer to the criticism against K41 raised by Landau. In other words, the energy dissipation rate $\varepsilon_{n}$ can take various values even for the eddies with the same diameter. The distribution of the values, i.e., the distribution of $\alpha$, is determined by a delicate balance between the non-linear convective term and the dissipative term in the N-S equation.

\section{Distribution of $\alpha$ and multifractal spectrum}

\subsection{Framework of multifractal analysis}

One needs $\left(\ell_{n} / \ell_{0}\right)^{-d}$ boxes in order to cover whole the space of volume $1^{d}$ in $d$ dimensional space by the boxes with volume $\left(\ell_{n} / \ell_{0}\right)^{d}$ without vacancy. Under the assumption that the eddies specified by $\alpha$ occupy the space with the fractal dimension $f_{d}(\alpha)$, the probability $P^{(n)}(\alpha) d \alpha$ to find a value within the domain $\alpha \sim \alpha+d \alpha$, when we pay attention to one of the boxes with volume $\left(\ell_{n} / \ell_{0}\right)^{d}$ in $d$ dimensional space, is given by the proportion of the number $\left(\ell_{n} / \ell_{0}\right)^{-f_{d}(\alpha)}$ of boxes, occupying $f_{d}(\alpha)$ dimensional space without vacancy, to the number $\left(\ell_{n} / \ell_{0}\right)^{-d}$ of all the boxes, i.e.,

$$
P^{(n)}(\alpha) d \alpha=c_{2}(\alpha)\left(\frac{\ell_{n}}{\ell_{0}}\right)^{d-f_{d}(\alpha)} d \alpha
$$

The fractal dimension $f_{d}(\alpha)$ is called the multifractal spectrum. In the following, we will proceed the investigation assuming that the $\alpha$ dependence of the normalization coefficient $c_{2}(\alpha)$ is negligible.

The mass exponent $\tau_{d}(\bar{q})$ is introduced by means of the partition function [5]

$$
Z_{d}^{(n)} \equiv \sum_{\# \text { of boxes }}\left(\frac{\varepsilon_{n} \ell_{n}^{d}}{\epsilon \ell_{0}^{d}}\right)^{\bar{q}}=\sum_{\# \text { of boxes }}\left(\frac{\ell_{n}}{\ell_{0}}\right)^{(\alpha-1+d) \bar{q}} \propto\left(\frac{\ell_{n}}{\ell_{0}}\right)^{-\tau_{d}(\bar{q})}
$$

The summation with respect to the number of boxes may be translated into the integration with respect to $\alpha$ as

$$
Z_{d}^{(n)}=\int d \alpha \rho(\alpha)\left(\frac{\ell_{n}}{\ell_{0}}\right)^{(\alpha-1+d) \bar{q}-f_{d}(\alpha)} .
$$

Evaluating the integration with the help of the method of the steepest descent in the limit $\ell_{n} / \ell_{0} \rightarrow 0$,

\footnotetext{
${ }^{4}$ In practice, as the resolutions in experiments or numerical simulations are finite, it may be appropriate to interpret that the term singularity here means to take abnormally large values.
} 
i.e., $n \rightarrow \infty$, we obtain the relation ${ }^{5}$

$$
f_{d}(\alpha)-(\alpha-1+d) \bar{q}=\tau_{d}(\bar{q}), \quad \bar{q}=\frac{d f_{d}(\alpha)}{d \alpha},
$$

which constitute the Legendre transformation between $f_{d}(\alpha)$ and $\tau_{d}(\bar{q})$. We also have

$$
\alpha-1+d=-\frac{d \tau_{d}(\bar{q})}{d \bar{q}} .
$$

The generalized dimension (the Rényi dimension) $D_{d}(\bar{q})$ is introduced by the relation

$$
\tau_{d}(\bar{q})=(1-\bar{q}) D_{d}(\bar{q})
$$

The $\bar{q}$ th moment of the energy dissipation rate is given by using the mass exponent as [5]

$$
\left\langle\left(\frac{\varepsilon_{n}}{\epsilon}\right)^{\bar{q}}\right\rangle \equiv \int_{0}^{\infty} d\left(\frac{\varepsilon_{n}}{\epsilon}\right)\left(\frac{\varepsilon_{n}}{\epsilon}\right)^{\bar{q}} P_{\epsilon}^{(n)}\left(\frac{\varepsilon_{n}}{\epsilon} \mid \varepsilon_{n} \neq 0\right) \sim\left(\frac{\ell_{n}}{\ell_{0}}\right)^{-\tau_{d}(\bar{q})+D_{d}-\bar{q} d}
$$

where the conditional probability $P_{\varepsilon}^{(n)}\left(\varepsilon_{n} / \epsilon \mid \varepsilon_{n} \neq 0\right) d\left(\varepsilon_{n} / \epsilon\right)$ to find in the box a non-zero value $\varepsilon_{n} / \epsilon$ between the domain $\varepsilon_{n} / \epsilon \sim \varepsilon_{n} / \epsilon+d\left(\varepsilon_{n} / \epsilon\right)$ is related to the probability $P^{(n)}(\alpha) d \alpha$ by

$$
P^{(n)}(\alpha) d \alpha=P_{\epsilon}^{(n)}\left(\frac{\varepsilon_{n}}{\epsilon} \mid \varepsilon_{n} \neq 0\right) d\left(\frac{\varepsilon_{n}}{\epsilon}\right) P_{\varepsilon_{n} \neq 0}^{(n)} .
$$

Here, $P_{\varepsilon_{n} \neq 0}^{(n)}$ is the probability that the selected box satisfies the condition $\varepsilon_{n} \neq 0$. Note that, for $\varepsilon_{n} \neq 0$, $\alpha$ is related with $\varepsilon_{n}$ by $\alpha=1+\ln \left(\varepsilon_{n} / \epsilon\right) / \ln \left(\ell_{n} / \ell_{0}\right)$ (see (10)). Assuming that the boxes satisfying $\varepsilon_{n} \neq 0$ occupy $D_{d}$ dimensional space without vacancy, we estimated $P_{\varepsilon_{n} \neq 0}^{(n)}$ as the proportion of the number $\left(\ell_{n} / \ell_{0}\right)^{-D_{d}}$ of boxes satisfying $\varepsilon_{n} \neq 0$ to the number $\left(\ell_{n} / \ell_{0}\right)^{-d}$ of all the boxes, i.e.,

$$
P_{\varepsilon_{n} \neq 0}^{(n)}=c_{1}\left(\frac{\ell_{n}}{\ell_{0}}\right)^{d-D_{d}}
$$

The condition for the normalization of probability, i.e., $\langle 1\rangle=1$, reduces to

$$
\tau_{d}(0)=D_{d}=D_{d}(\bar{q}=0)=f_{d}\left(\alpha_{\bar{q}=0}\right) .
$$

Whereas, the energy conservation law, i.e., $\left\langle\varepsilon_{n}\right\rangle=\epsilon$, reduces to

$$
\tau_{d}(1)=D_{d}-d \text {. }
$$

Since $\tau_{d}(1)=0$ in general when $D_{d}(1)$ is finite, $(22)$ gives

$$
D_{d}=D_{d}(\bar{q}=0)=d .
$$

The definition of the intermittency exponent $\mu$, i.e.,

$$
\left\langle\left(\frac{\varepsilon_{n}}{\epsilon}\right)^{2}\right\rangle=\left(\frac{\ell_{n}}{\ell_{0}}\right)^{-\mu}
$$

provides us with

$$
\mu=\tau_{d}(2)-D_{d}+2 d .
$$

Summarizing (21), (23) and (25), we have

$$
\tau_{d}(0)=D_{d}(\bar{q}=0)=d, \quad \tau_{d}(1)=0, \quad \mu=d+\tau_{d}(2)=d-D_{d}(2) .
$$

Note that the first equation in (26) is also obtained by noticing that the number of boxes with the side length $\ell_{n}$ necessary to cover the $d$ dimensional space is given by $\sum_{\# \text { of boxes }} 1 \propto\left(\ell_{n} / \ell_{0}\right)^{-d}$, and that $\sum_{\text {\# of boxes }} 1 \propto\left(\ell_{n} / \ell_{0}\right)^{-\tau_{d}(0)}$ from (13), i.e., the definition of $\tau_{d}(\bar{q})$. Notice that the scaling exponent of the $m$ th order velocity structure function defined by (11) is related to the mass exponent as

$$
\zeta_{m}=d-\tau_{d}\left(\frac{m}{3}\right)+(1-d) \frac{m}{3}
$$

\footnotetext{
${ }^{5}$ We are neglecting the $\alpha$ dependence of the density $\rho(\alpha)$ introduced in the translation $\sum_{\# \text { of boxes }}=$ $\int d \alpha \rho(\alpha)\left(\ell_{n} / \ell_{0}\right)^{-f_{d}(\alpha)}$.
} 


\subsection{Reduced representation}

Introducing $f(\alpha)$ and $\tau(\bar{q})$, respectively, through the relations

$$
f_{d}(\alpha)=f(\alpha)+d-1, \quad \tau_{d}(\bar{q})=\tau(\bar{q})+(d-1)(1-\bar{q}),
$$

and $D(\bar{q})$ by

$$
\tau(\bar{q})=(1-\bar{q}) D(\bar{q}),
$$

the Legendre transformation, consisting of (15) and (16), reduces to

$$
f(\alpha)=\alpha \bar{q}+\tau(\bar{q}), \quad \bar{q}=\frac{d f(\alpha)}{d \alpha}, \quad \alpha=-\frac{d \tau(\bar{q})}{d \bar{q}},
$$

and the relations in (26) and the one in (27), respectively, to

$$
\tau(0)=D(0)=f\left(\alpha_{0}\right)=1, \quad \tau(1)=0, \quad \mu=1+\tau(2)=1-D(2)
$$

and

$$
\zeta_{m}=1-\tau\left(\frac{m}{3}\right)
$$

Then, the probability (12) reduces to

$$
P^{(n)}(\alpha) d \alpha \propto\left(\frac{\ell_{n}}{\ell_{0}}\right)^{1-f(\alpha)} d \alpha .
$$

Note that $D_{d}(\bar{q})$ and $D(\bar{q})$ are related with each other by

$$
D_{d}(\bar{q})=D(\bar{q})+d-1 .
$$

It may be worthwhile to note here that the system with $f(\alpha), \tau(\bar{q})$ and $D(\bar{q})$ is not only for the analyses of multifractal structure of data-set with $d=1$ but also for those with general dimension $d>1$. The (multi)-fractal structures in $d$-dim are accumulated altogether into the (multi)-fractal dimension $f(\alpha)=$ $f_{d}(\alpha)-(d-1)$.

\subsection{Log-normal model as a multifractal system}

Within the log-normal model $[22,23,24]$, the ratios $\varepsilon_{j} / \varepsilon_{j-1}(j=1,2, \cdots)$ of the energy dissipation rates are regarded as stochastic variables defined within the domain $[0, \infty]$. This ratio provides a measure how much energy received by the eddy with the diameter $\ell_{j-1}$ is delivered to the eddy of the size $\ell_{j}$. We see that the summation of the stochastic variables $\ln \left(\varepsilon_{j} / \varepsilon_{j-1}\right)$ is related to $\alpha$ by the relation

$$
\frac{1}{\sqrt{n \sigma^{2}}} \sum_{j=1}^{n} \ln \left(\frac{\varepsilon_{j}}{\varepsilon_{j-1}}\right)=\frac{\sqrt{n}}{\sigma}(1-\alpha) \ln \delta .
$$

Assuming that the variables $\ln \left(\varepsilon_{j} / \varepsilon_{j-1}\right)(j=1,2, \cdots)$ are stochastically independent, and that they have an identical distribution function, one concludes that the distribution of the stochastic variables must be the canonical distribution (Gaussian distribution)

$$
P^{(n)}(\alpha) \propto \mathrm{e}^{-n\left(\alpha-\alpha_{0}\right)^{2} / 2 \sigma^{2}}
$$

because of the central limit theorem for $n \gg 1$. The domain of $\alpha$ is $[-\infty, \infty]$.

Substituting (36) into the left-hand side of (33), we have

$$
f(\alpha)=1-\frac{\left(\alpha-\alpha_{0}\right)^{2}}{2 \sigma^{2} \ln \delta}=1-\frac{(\alpha-1-\mu / 2)^{2}}{2 \mu} .
$$

At the second equality, we have used

$$
\alpha_{0}=1+\frac{\mu}{2}, \quad \sigma^{2}=\frac{\mu}{\ln \delta}
$$


which are derived with the help of the two independent relations, i.e., the energy conservation law given by the second equation in (31) and the definition of the intermittency exponent $\mu$ given by the third equation in (31). Note that the final expression of $f(\alpha)$ in (37) does not depend on $\delta$ nor on $n$. The mass exponent has the form

$$
\tau(\bar{q})=(1-\bar{q})\left(1-\frac{\mu \bar{q}}{2}\right)
$$

which is derived through the Legendre transformation (15). Therefore, the generalized dimension for the log-normal model becomes $D_{\bar{q}}=1-\mu \bar{q} / 2$.

Substituting (38) into (36), we have

$$
P^{(n)}(\alpha) \propto \mathrm{e}^{-\tilde{n}(\alpha-1-\mu / 2)^{2} / 2 \mu}
$$

with (3) for $\tilde{n}$, which does not depend on the magnification $\delta$. Note that $\mu$ should not depend on $\delta$ since $\mu$ is unique once a turbulent system is specified, and that the dependence of $P^{(n)}(\alpha)$ on $\delta$ is absorbed into $\sigma^{2}$ with the help of $\tilde{n}$. It may be reasonable to interpret $\tilde{n}$ as the "renormalized" number of stages in the cascade model.

\section{Theoretical framework of MPDFT}

\subsection{A\&A model}

For $P^{(n)}(\alpha)$, A\&A model within MPDFT $[10,11,12,13,15,16,18,19,21]$ adopts the Tsallis-type distribution function

$$
P^{(n)}(\alpha) \propto\left[1-\frac{\left(\alpha-\alpha_{0}\right)^{2}}{(\Delta \alpha)^{2}}\right]^{n /(1-q)}
$$

with

$$
\Delta \alpha=\sqrt{\frac{2 X}{(1-q) \ln \delta}} .
$$

Here, $q$ is the entropy index appeared in the extensive Rényi entropy [27] and the non-extensive HCT entropy [28, 29]. With the common constraints 1) Conservation of probability with respect to the linear PDF for $\alpha$ and 2) Fixing the normalized $q$-variance, the MaxEnt PDFs for both entropies have the same functional structure, i.e., this Tsallis-type distribution function. ${ }^{6}$ The domain of $\alpha$ is $\alpha_{\min } \leq \alpha \leq \alpha_{\max }$ where $\alpha_{\min }$ and $\alpha_{\max }$ are given by $\alpha_{\min , \max }=\alpha_{0} \mp \Delta \alpha$. The proportional coefficient of (41) is specified by the normalization condition

$$
\int_{\alpha_{\min }}^{\alpha_{\max }} d \alpha P^{(n)}(\alpha)=1
$$

The substitution of (41) into the left-hand side of (33) gives, for $n \gg 1$, the multifractal spectrum

$$
f(\alpha)=1+\frac{1}{(1-q) \ln \delta} \ln \left[1-\frac{\left(\alpha-\alpha_{0}\right)^{2}}{(\Delta \alpha)^{2}}\right] .
$$

The mass exponent has the form

$$
\tau(\bar{q})=1-\alpha_{0} \bar{q}+\frac{2 X \bar{q}^{2}}{1+\sqrt{C_{\bar{q}}}}+\frac{\ln 2}{(1-q) \ln \delta}\left[1-\log _{2}\left(1+\sqrt{C_{\bar{q}}}\right)\right]
$$

with

$$
C_{\bar{q}}=1+2 \bar{q}^{2}(1-q) X \ln \delta .
$$

The three parameters $\alpha_{0}, X$ and $q$ are determined as the functions of $\mu$ with the help of the three conditions, i.e., the energy conservation law given by the second equation in (31), the definition of the

\footnotetext{
${ }^{6}$ Recently there are attractive developments [33, 34] related to the MaxEnt PDFs for the Rényi and the HCT entropies. It may be one of the attractive future problems to investigate these developments in terms of MPDFT with the help of the turbulent data provided by DNSs or by experiments. It will be given elsewhere in the near future.
} 
intermittency exponent given by the third equation in (31), and the scaling relation (2) with $\alpha_{ \pm}$being the solution of $f\left(\alpha_{ \pm}\right)=0$ :

$$
\alpha_{ \pm}=\alpha_{0} \pm \sqrt{2 b X}
$$

where

$$
b=\frac{1-\mathrm{e}^{-(1-q) \ln \delta}}{(1-q) \ln \delta} .
$$

The scaling relation (2) is a generalization of the one introduced by Tsallis and others [25, 26] to which (2) reduces, when $\delta=2$. This generalization was born out of the theoretical framework, A\&A model within MPDFT, itself. The new scaling relation (2) is solved to give

$$
\sqrt{2 b X}=-(1-q) \log _{2} \delta+\sqrt{\alpha_{0}^{2}+\left[(1-q) \log _{2} \delta\right]^{2}} \text {. }
$$

The parameter $q$ is determined, altogether with $\alpha_{0}$ and $X$, as a function of $\mu$ only in the combination $(1-q) \ln \delta$. It is quite reasonable in the following reason. The value of the magnification $\delta$ is determined arbitrarily by observers, therefore its value should not affect the values of physical quantities as long as one studies a turbulent system. The difference in $\delta$ is absorbed into the entropy index $q$, which may be reasonable in the sense that changing the zooming rate $\delta$ may result in picking up the different hierarchy, containing the entropy specified by the index $q$, out of multifractal structure of turbulence. The detailed investigation of this point will be given elsewhere. With the above generalization of the scaling relation, as the parameters are dependent on $q$ only in the combination $(1-q) \ln \delta$, we are naturally lead to the replacement of $n$ in the expression of $P^{(n)}(\alpha)$ in (41) with $\tilde{n}$ defined by (3), i.e.,

$$
P^{(n)}(\alpha) \propto\left[1-\frac{\left(\alpha-\alpha_{0}\right)^{2}}{(\Delta \alpha)^{2}}\right]^{\tilde{n} /(1-q) \ln \delta} .
$$

If we assume that $\tilde{n}$ does not depend on $\delta$, the distribution of $\alpha$ itself becomes also independent of $\delta$. Then, A\&A model within MPDFT has come to the framework in which the choice of the magnification $\delta$ does not affect the PDF for $\alpha$ in addition to observables. Note that the new scaling relation (2) is found to be intimately related to $\delta$-scale Cantor sets produced by a series of the unstable $\delta^{\infty}$ periodic orbits. $\tilde{n}$ may be interpreted as the number of stages in the $\delta$-scale Cantor set, and can be understood, intuitively, as the number of stages in the energy cascade model.

\subsection{PDFs for observables}

Now, we derive the general formula for PDFs within MPDFT for an observable

$$
\left|x^{\prime}\right|=\lim _{n \rightarrow \infty}\left|x_{n}^{\prime}\right|, \quad\left|x_{n}^{\prime}\right|=\left(\frac{\delta x_{n}}{\ell_{n}}\right)\left(\frac{\delta x_{0}}{\ell_{0}}\right)^{-1}
$$

representing intermittent singular behavior, where $\delta x_{n}=\left|x\left(\bullet+\ell_{n}\right)-x(\bullet)\right|$ is the fluctuation of a physical quantity $x$, and is assumed to be related to $\alpha$ through

$$
\left|x_{n}\right|=\frac{\delta x_{n}}{\delta x_{0}}=\left(\frac{\ell_{n}}{\ell_{0}}\right)^{\phi \alpha / 3} .
$$

Here the parameter $\phi$ is determined once the physical field $x(\bullet)$ is specified (see below). Since

$$
\left|x_{n}^{\prime}\right|=\left|x_{n}\right|\left(\frac{\ell_{n}}{\ell_{0}}\right)^{-1}=\left(\frac{\ell_{n}}{\ell_{0}}\right)^{(\phi \alpha / 3)-1},
$$

$\left|x^{\prime}\right|$ diverges for $\alpha<3 / \phi$. Note that $\left|x^{\prime}\right|$ reduces to the velocity derivative and fluid particle acceleration for $\phi=1$ and $\phi=2$, respectively, and formally to the energy dissipation rates (10) for $\phi=3$. The probability $\Pi_{\phi}^{\prime(n)}\left(x_{n}^{\prime}\right) d x_{n}^{\prime}$ to find the physical quantity $x_{n}^{\prime}$ taking a value in the domain $x_{n}^{\prime} \sim x_{n}^{\prime}+d x_{n}^{\prime}$ is 
supposed to be rephrased by the probability $\Pi_{\phi}^{(n)}\left(x_{n}\right) d x_{n}$ to find the physical quantity $x_{n}$ taking a value in the domain $x_{n} \sim x_{n}+d x_{n}$ with the relation

$$
\Pi_{\phi}^{\prime(n)}\left(x_{n}^{\prime}\right) d x_{n}^{\prime}=\Pi_{\phi}^{(n)}\left(x_{n}\right) d x_{n} .
$$

We are assuming that the PDF $\Pi_{\phi}^{(n)}\left(x_{n}\right)$ can be divided into two parts as

$$
\Pi_{\phi}^{(n)}\left(x_{n}\right)=\Pi_{\phi, \mathrm{S}}^{(n)}\left(x_{n}\right)+\Delta \Pi_{\phi}^{(n)}\left(x_{n}\right) .
$$

The first term describes the contribution from the abnormal part of the physical quantity $x_{n}$ due to the fact that its singularities distribute themselves multifractal way in real space. It is the part given by

$$
\Pi_{\phi, \mathrm{S}}^{(n)}\left(\left|x_{n}\right|\right) d\left|x_{n}\right|=\bar{\Pi}_{\phi, \mathrm{S}}^{(n)} P^{(n)}(\alpha) d \alpha
$$

through the variable translation (52) between $\left|x_{n}\right|$ and $\alpha$. The second term $\Delta \Pi_{\phi}^{(n)}\left(x_{n}\right)$ represents the contributions from the dissipative term in the N-S equation and/or from the errors in measurements, etc., and is interpreted as a correction to the first term.

The dissipative term violates the invariance based on the scale transformation (5), and, therefore, the effect of dissipation has been neglected in the consideration of $\Pi_{\phi, S}^{(n)}\left(x_{n}\right)$ for the distribution of singularities. The values of $\left|x_{n}\right|$ representing the part originated from the singularities describe the part larger than the standard deviation. Whereas, the values of $\left|x_{n}\right|$ for the part contributing to the correction term are smaller than or about the order of its standard deviation.

\subsection{PDFs for variables taking positive values}

Let us consider the PDF for the variable $x_{n}^{\prime}$ whose range is $\left(0, \Lambda_{n}\right)$. The $m$ th moment of $x_{n}^{\prime}$ is translated into the $m$ th moment of the structure functions for the variable $x_{n}$, i.e.,

$$
\left.\int_{0}^{\Lambda_{n}} d x_{n}^{\prime}\left(x_{n}^{\prime}\right)^{m} \Pi_{\phi}^{\prime(n)}\left(x_{n}^{\prime}\right)=\left(\frac{\ell_{n}}{\ell_{0}}\right)^{-m}\left\langle\left(x_{n}\right)^{m}\right\rangle\right\rangle
$$

where

$$
\left\langle\left\langle\left(x_{n}\right)^{m}\right\rangle\right\rangle=\int_{0}^{\Lambda_{n}} d x_{n}\left(x_{n}\right)^{m} \Pi_{\phi}^{(n)}\left(x_{n}\right)
$$

with

$$
\Lambda_{n}=\left(\frac{\ell_{n}}{\ell_{0}}\right)^{\left(\phi \alpha_{\min } / 3\right)-1} .
$$

The normalization of $\Pi_{\phi}^{\prime(n)}\left(x_{n}^{\prime}\right)$ is given by

$$
\int_{0}^{\Lambda_{n}} d x_{n}^{\prime} \Pi_{\phi}^{\prime(n)}\left(x_{n}^{\prime}\right)=\langle\langle 1\rangle\rangle=1 .
$$

Substituting (55) with (56) into (58), we have

$$
\begin{aligned}
\left.\left\langle\left(x_{n}\right)^{m}\right\rangle\right\rangle & =\int_{0}^{\Lambda_{n}} d x_{n}\left(x_{n}\right)^{m} \Pi_{\phi, \mathrm{S}}^{(n)}\left(x_{n}\right)+\gamma_{\phi, m}^{(n)} \\
& =\bar{\Pi}_{\phi, \mathrm{S}}^{(n)} \int_{\alpha_{\min }}^{\alpha_{\max }} d \alpha\left(\frac{\ell_{n}}{\ell_{0}}\right)^{\phi m \alpha / 3} P^{(n)}(\alpha)+\gamma_{\phi, m}^{(n)} \\
& =\bar{\Pi}_{\phi, \mathrm{S}}^{(n)} a_{\phi m}\left(\frac{\ell_{n}}{\ell_{0}}\right)^{1-\tau(\phi m / 3)}+\gamma_{\phi, m}^{(n)}
\end{aligned}
$$

with

$$
\gamma_{\phi, m}^{(n)}=\int_{0}^{\Lambda_{n}} d x_{n}\left(x_{n}\right)^{m} \Delta \Pi_{\phi}^{(n)}\left(x_{n}\right),
$$




$$
a_{\phi m}=\sqrt{\frac{\left|f^{\prime \prime}\left(\alpha_{0}\right)\right|}{\left|f^{\prime \prime}\left(\alpha_{\phi m / 3}\right)\right|}}
$$

where $f^{\prime \prime}(\alpha)=d^{2} f(\alpha) / d \alpha^{2}$. The third equality in (61) is estimated for $n \gg 1$. For $m=0$, (61) gives $\langle\langle 1\rangle\rangle=1$ with the normalization condition (60), hence we have

$$
\bar{\Pi}_{\phi, \mathrm{S}}^{(n)}=1-\gamma_{\phi, 0}^{(n)} .
$$

Here, we used the facts $a_{0}=1$ and $\tau(0)=1$. We finally obtain the compact expression for the $m$ th moment of the structure function in the form

$$
\left\langle\left(x_{n}\right)^{m}\right\rangle=\gamma_{\phi, m}^{(n)}+\left(1-\gamma_{\phi, 0}^{(n)}\right) a_{\phi m}\left(\frac{\ell_{n}}{\ell_{0}}\right)^{\zeta_{\phi m}}
$$

with the corresponding scaling exponents

$$
\zeta_{\phi m}=1-\tau\left(\frac{\phi m}{3}\right) .
$$

For $\phi=1, \zeta_{\phi m}$ reduces to the scaling exponents $\zeta_{m}$ of the $m$ th order velocity structure function defined by (11). Note that $\Pi_{\phi, \mathrm{S}}^{(n)}\left(x_{n}\right) d x_{n}$ now has the compact form

$$
\Pi_{\phi, \mathrm{S}}^{(n)}\left(\left|x_{n}\right|\right) d\left|x_{n}\right|=\left(1-\gamma_{\phi, 0}^{(n)}\right) \sqrt{\frac{\left|f^{\prime \prime}\left(\alpha_{0}\right)\right|\left|\ln \left(\ell_{n} / \ell_{0}\right)\right|}{2 \pi}}\left(\frac{\ell_{n}}{\ell_{0}}\right)^{1-f(\alpha)} d \alpha .
$$

It is convenient to introduce the PDF

$$
\hat{\Pi}_{\phi}^{(n)}\left(\xi_{n}\right) d \xi_{n}=\Pi_{\phi}^{(n)}\left(x_{n}^{\prime}\right) d x_{n}^{\prime}
$$

for the variable

$$
\xi_{n}=\frac{x_{n}^{\prime}}{\sqrt{\left\langle\left\langle\left(x_{n}^{\prime}\right)^{2}\right\rangle_{\mathrm{c}}\right.}}=\frac{x_{n}}{\sqrt{\left\langle\left\langle\left(x_{n}\right)^{2}\right\rangle\right\rangle_{\mathrm{c}}}}
$$

normalized by their own standard deviations with

$$
\left\langle\left\langle\left(x_{n}\right)^{2}\right\rangle\right\rangle_{c}=\left\langle\left\langle\left(x_{n}\right)^{2}\right\rangle\right\rangle-\left\langle\left\langle x_{n}\right\rangle\right\rangle^{2} .
$$

The normalized variable $\xi_{n}$ is related to $\alpha$ through the relation

$$
\xi_{n}=\bar{\xi}_{n}\left(\frac{\ell_{n}}{\ell_{0}}\right)^{(\phi \alpha / 3)-\zeta_{2 \phi} / 2}
$$

with

$$
\bar{\xi}_{n}=\left[\left\{\gamma_{\phi, 2}^{(n)}-\left[\gamma_{\phi, 1}^{(n)}+\left(1-\gamma_{\phi, 0}^{(n)}\right) a_{\phi} \delta_{n}^{\zeta_{\phi}}\right]^{2}\right\} \delta_{n}^{-\zeta_{2 \phi}}+\left(1-\gamma_{\phi, 0}^{(n)}\right) a_{2 \phi}\right]^{-1 / 2} .
$$

It is reasonable to assume that the origin of intermittent rare events is attributed to the first singular term in (55), and that the contribution from the second term $\Delta \Pi_{\phi}^{(n)}\left(x_{n}\right)$ to the events is negligible. Therefore, we put, for $\xi_{n}^{*} \leq \xi_{n} \leq \xi_{n}^{\max }$ (equivalently, $\alpha_{\min } \leq \alpha \leq \alpha^{*}$ ),

$$
\hat{\Pi}_{\phi, \mathrm{tl}}^{(n)}\left(\xi_{n}\right) d \xi_{n}=\Pi_{\phi, \mathrm{S}}^{(n)}\left(x_{n}\right) d x_{n},
$$

where

$$
\xi_{n}^{\max }=\bar{\xi}_{n}\left(\frac{\ell_{n}}{\ell_{0}}\right)^{\left(\phi \alpha_{\min } / 3\right)-\zeta_{2 \phi} / 2} .
$$

Substituting (56) with (33) into (73), we have

$$
\hat{\Pi}_{\phi, \mathrm{tl}}^{(n)}\left(\xi_{n}\right)=\bar{\Pi}_{\phi}^{(n)}\left(\frac{\ell_{n}}{\ell_{0}}\right)^{\left(\zeta_{2 \phi} / 2\right)-(\phi \alpha / 3)+1-f(\alpha)}=\bar{\Pi}_{\phi}^{(n)}\left(\frac{\ell_{n}}{\ell_{0}}\right)^{1-f(\alpha)} \frac{\bar{\xi}_{n}}{\xi_{n}}
$$


with

$$
\bar{\Pi}_{\phi}^{(n)}=\frac{3\left(1-\gamma_{\phi, 0}^{(n)}\right)}{\phi \bar{\xi}_{n}} \sqrt{\frac{\left|f^{\prime \prime}\left(\alpha_{0}\right)\right|}{2 \pi\left|\ln \delta_{n}\right|}} .
$$

Note that $\xi_{n}^{*} \simeq 1$ as can be seen below when we analyze experiments. This tail part $\hat{\Pi}_{\phi, \mathrm{tl}}^{(n)}\left(\left|\xi_{n}\right|\right)$ represents the large deviations, and manifests itself the multifractal distribution of the singularities due to the scale invariance of the Navier-Stokes equation when its dissipative term can be neglected.

For smaller values, $\left|\xi_{n}\right| \leq \xi_{n}^{*}$ (equivalently, $\alpha^{*} \leq \alpha$ ), we put

$$
\hat{\Pi}_{\phi, \mathrm{cr}}^{(n)}\left(\xi_{n}\right) d \xi_{n}=\left[\Pi_{\phi, \mathrm{S}}^{(n)}\left(x_{n}\right)+\Delta \Pi_{\phi}^{(n)}\left(x_{n}\right)\right] d x_{n}=\bar{\Pi}_{\phi, \mathrm{cr}}^{(n)} \mathrm{e}^{-g_{\phi}\left(\xi_{n}\right)} d \xi_{n}
$$

with some real valued function $g_{\phi}\left(\xi_{n}\right)$. The contribution to this center part $\hat{\Pi}_{\phi, \mathrm{cr}}^{(n)}\left(\xi_{n}\right)$ comes, mainly, from thermal fluctuations.This part is responsible to smaller fluctuations of the variable, compared with its standard deviation, stemmed from the dissipative term violating the scale invariance.

The two parts of the PDF, $\hat{\Pi}_{\phi, \mathrm{tl}}^{(n)}\left(\xi_{n}\right)$ and $\hat{\Pi}_{\phi, \mathrm{cr}}^{(n)}\left(\xi_{n}\right)$, are connected at

$$
\xi_{n}^{*}=\bar{\xi}_{n}\left(\frac{\ell_{n}}{\ell_{0}}\right)^{\left(\phi \alpha^{*} / 3\right)-\zeta_{2 \phi} / 2}
$$

under the conditions that they have the common value, i.e.,

$$
\hat{\Pi}_{\phi, \mathrm{tl}}^{(n)}\left(\xi_{n}^{*}\right)=\hat{\Pi}_{\phi, \mathrm{cr}}^{(n)}\left(\xi_{n}^{*}\right)
$$

and the common log-slope, i.e.,

$$
\left.\frac{d}{d \xi_{n}} \ln \hat{\Pi}_{\phi, \mathrm{tl}}^{(n)}\left(\xi_{n}\right)\right|_{\xi_{n}=\xi_{n}^{*}}=\left.\frac{d}{d \xi_{n}} \ln \hat{\Pi}_{\phi, \mathrm{cr}}^{(n)}\left(\xi_{n}\right)\right|_{\xi_{n}=\xi_{n}^{*}} .
$$

The conditions (79) and (80) give

$$
\bar{\Pi}_{\phi, \mathrm{cr}}^{(n)}=\bar{\Pi}_{\phi}^{(n)} \mathrm{e}^{g_{\phi}\left(\xi_{n}^{\star}\right)}\left(\frac{\ell_{n}}{\ell_{0}}\right)^{1-f\left(\alpha^{\star}\right)} \frac{\bar{\xi}_{n}}{\bar{\xi}_{n}^{*}}
$$

and

$$
\left.\frac{d g_{\phi}\left(\xi_{n}\right)}{d \xi_{n}}\right|_{\xi_{n}=\xi_{n}^{*}}=\frac{\phi+3 f^{\prime}\left(\alpha^{*}\right)}{\phi \xi_{n}^{*}}
$$

respectively. With the help of (81), we obtain

$$
\hat{\Pi}_{\phi, \mathrm{cr}}^{(n)}\left(\xi_{n}\right)=\bar{\Pi}_{\phi}^{(n)} \mathrm{e}^{-\left[g_{\phi}\left(\xi_{n}\right)-g_{\phi}\left(\xi_{n}^{*}\right)\right]}\left(\frac{\ell_{n}}{\ell_{0}}\right)^{1-f\left(\alpha^{*}\right)} \frac{\bar{\xi}_{n}}{\xi_{n}^{*}} .
$$

The value of $\alpha^{*}$, therefore the value of $\xi_{n}^{*}$ (see (78)), is determined for each PDF as an adjusting parameter in the analysis of PDFs obtained by ordinary or numerical experiments. ${ }^{7}$ By this refinement, the PDF $\hat{\Pi}_{\phi}^{(n)}\left(\xi_{n}\right)$ has come to be able to reproduce experimental PDFs around the connection point much better than before with only negligible changes at the tail part and the center part of the PDF. Note that the reproduction of the PDF for the tail and central parts within MPDFT have been quite satisfactory from the beginning.

With the assumption (73) that, for $\xi_{n}^{*} \leq \xi_{n} \leq \xi_{n}^{\max }$, one can neglect the contribution from $\Delta \Pi_{\phi}^{(n)}\left(x_{n}\right) d x_{n}$, we are able to write down the formula to calculate $2 \gamma_{\phi, m}^{(n)}$. From (77), (83) and (67), $\Delta \Pi_{\phi}^{(n)}\left(x_{n}\right) d x_{n}$ has its value

$$
\begin{aligned}
\Delta \Pi_{\phi}^{(n)}\left(x_{n}\right) d x_{n}= & \bar{\Pi}_{\phi}^{(n)} \mathrm{e}^{-\left[g_{\phi}\left(\xi_{n}\right)-g_{\phi}\left(\xi_{n}^{*}\right)\right]}\left(\frac{\ell_{n}}{\ell_{0}}\right)^{1-f\left(\alpha^{\star}\right)} \frac{\bar{\xi}_{n}}{\xi_{n}^{*}} d \xi_{n} \\
& -\left(1-\gamma_{\phi, 0}^{(n)}\right) \sqrt{\frac{\left|f^{\prime \prime}\left(\alpha_{0}\right)\right|\left|\ln \left(\ell_{n} / \ell_{0}\right)\right|}{2 \pi}}\left(\frac{\ell_{n}}{\ell_{0}}\right)^{1-f(\alpha)} d \alpha
\end{aligned}
$$

${ }^{7}$ In the previous treatments, the connection point $\xi_{n}^{*}$ had been determined as the point where $\hat{\Pi}_{\phi}^{(n)}\left(\xi_{n}^{*}\right)$ has the least dependence on $n$. 
only for the center part, hence, from (62), we have

$$
\gamma_{\phi, m}^{(n)}=\int_{0}^{x_{n}^{*}} d x_{n}\left(x_{n}\right)^{m} \Delta \Pi_{\phi}^{(n)}\left(x_{n}\right)=\left(1-\gamma_{\phi, 0}^{(n)}\right)\left(K_{\phi, m}^{(n)}-L_{\phi, m}^{(n)}\right)
$$

with

$$
\begin{aligned}
K_{\phi, m}^{(n)} & =\frac{3}{\phi}\left(\frac{\ell_{n}}{\ell_{0}}\right)^{1-f\left(\alpha^{*}\right)+m \phi \alpha^{*} / 3} \sqrt{\frac{\left|f^{\prime \prime}\left(\alpha_{0}\right)\right|}{2 \pi\left|\ln \left(\ell_{n} / \ell_{0}\right)\right|}} \int_{0}^{1} d z z^{m} \mathrm{e}^{-\left[g_{\phi}\left(\xi_{n}^{*} z\right)-g_{\phi}(1)\right]}, \\
L_{\phi, m}^{(n)} & =\left(\frac{\ell_{n}}{\ell_{0}}\right) \sqrt{\frac{\left|f^{\prime \prime}\left(\alpha_{0}\right)\right|\left|\ln \left(\ell_{n} / \ell_{0}\right)\right|}{2 \pi}} \int_{\alpha^{*}}^{\alpha_{\max }} d \alpha\left(\frac{\ell_{n}}{\ell_{0}}\right)^{m \alpha \phi / 3-f(\alpha)} .
\end{aligned}
$$

For $m=0,(85)$ gives

$$
1-\gamma_{\phi, 0}^{(n)}=\frac{1}{1+K_{\phi, 0}^{(n)}-L_{\phi, 0}^{(n)}}
$$

Then, we finally obtain

$$
\gamma_{\phi, m}^{(n)}=\frac{K_{\phi, m}^{(n)}-L_{\phi, m}^{(n)}}{1+K_{\phi, 0}^{(n)}-L_{\phi, 0}^{(n)}} .
$$

The flatness $F_{x}^{(n)}$ of the PDF for the variable $x_{n}$, and therefore of the PDF for $x_{n}^{\prime}$, defined by

$$
F_{x}^{(n)}=\frac{\left\langle\left\langle\left|x_{n}\right|^{4}\right\rangle\right\rangle}{\left\langle\left\langle\left|x_{n}\right|^{2}\right\rangle\right\rangle^{2}}=\left\langle\left\langle\xi_{n}^{4}\right\rangle\right\rangle
$$

reduces to

$$
F_{x}^{(n)}=\frac{\gamma_{\phi, 4}^{(n)}+\left(1-\gamma_{\phi, 0}^{(n)}\right) a_{4 \phi}\left(\ell_{n} / \ell_{0}\right)^{\zeta_{4 \phi}}}{\left[\gamma_{\phi, 2}^{(n)}+\left(1-\gamma_{\phi, 0}^{(n)}\right) a_{2 \phi}\left(\ell_{n} / \ell_{0}\right)^{\zeta_{2 \phi}}\right]^{2}}
$$

\section{Analyses of observed PDFs for energy dissipation rates}

In order to see that the value $\delta$ actually does not affect the values of observables within the framework of MPDFT, we will analyze the PDFs of energy dissipation rates extracted by Kaneda's group from their $4096^{3}$ DNS [30]. The theoretical PDF for energy dissipation rates is given by the formulae in subsection 4.2 with $\phi=3$. We put for the center part of the $\mathrm{PDF}, \hat{\Pi}_{\phi, \mathrm{cr}}^{(n)}\left(\xi_{n}\right)$ in $(77)$, the trial function

$$
\mathrm{e}^{-g_{3}\left(\xi_{n}\right)}=\left(\frac{\xi_{n}}{\xi_{n}^{*}}\right)^{\theta-1}\left\{1-\left(1-q^{\prime}\right) \frac{1+f^{\prime}\left(\alpha^{*}\right)}{w}\left[\left(\frac{\xi_{n}}{\xi_{n}^{*}}\right)^{w}-1\right]\right\}^{1 /\left(1-q^{\prime}\right)}
$$

of the Tsallis-type with three parameters $w, q^{\prime}$ and $\theta$. The first parameter $w$ is settled to $w=1.40$ throughout the following analyses in this paper. It is adjusted by the property of the experimental PDFs around the peak point with the theoretical PDF $\hat{\Pi}_{\phi}^{(n)}\left(\xi_{n}\right)$. The second parameter $q^{\prime}$ is the entropy index different from $q$ in (41). The third parameter $\theta$ is determined by the property of PDF near $\xi_{n}=0$.

The PDFs of energy dissipation rates by DNS are analyzed in Fig. 1, Fig. 2 and Fig. 3, respectively, for the magnifications $\delta=2^{1 / 4}, \delta=2^{1 / 2}$ and $\delta=2$ on (a) $\log$ and (b) linear scale in the vertical axes. For better visibility, each PDF is shifted by appropriate unit along the vertical axis. Open circles in the figures are the DNS data points for PDF. Solid lines represent the theoretical PDFs given in subsection 4.2 with $\phi=3$.

Through the analyses, it turns out that the turbulent system under consideration is characterized with the value $\mu=0.345$ for the intermittency exponent. Then, the parameters necessary for the PDF within A\&A model are determined as $(1-q) \ln \delta=0.299, \alpha_{0}=1.20$ and $X=0.411$, which are independent of $\delta$. It may be reasonable to introduce the "renormalized" entropy index $q_{R}$ through the relation $1-q_{R}=(1-q) \ln \delta$. The value $q_{R}=0.701$ is common to all the cases $\delta=2^{1 / 4}, 2^{1 / 2}$ and 2 for the 

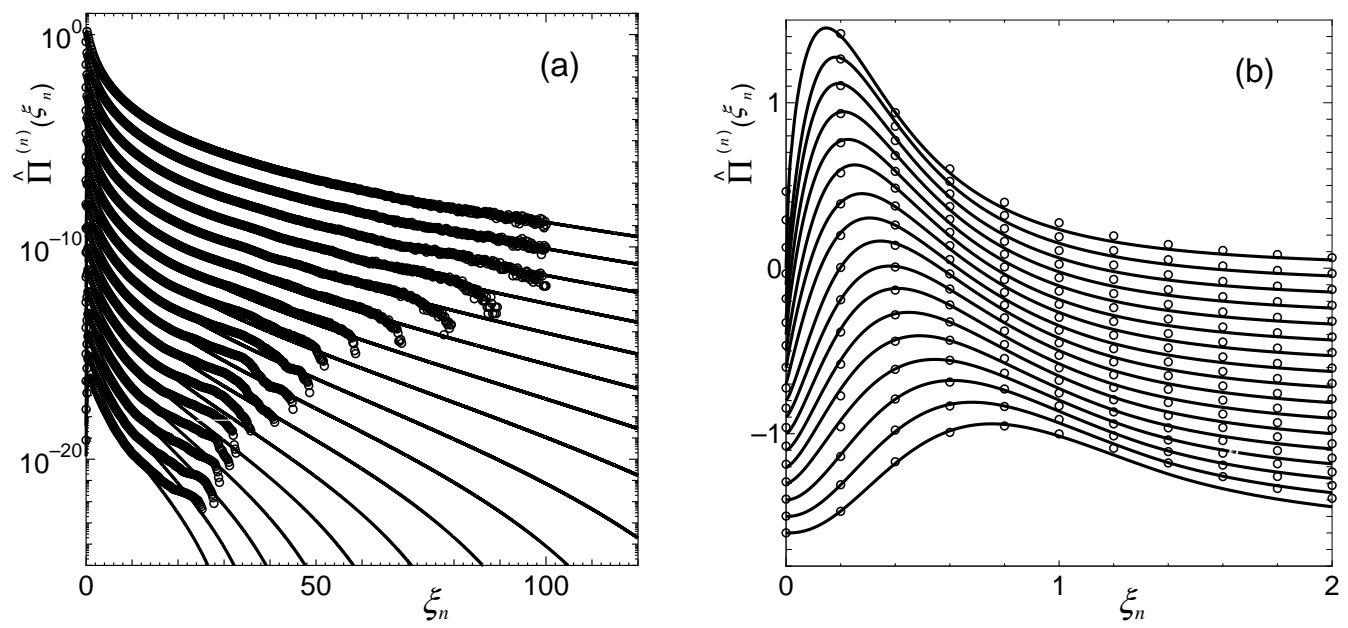

Figure 1: The PDFs of energy dissipation rates for $\delta=2^{1 / 4}$ on (a) log and (b) linear scale in the vertical axes. For better visibility, each PDF is shifted by -1 unit along the vertical axis in (a) and by -0.1 unit along the vertical axis in (b). Open circles are the PDFs by DNS from the smallest value (top) to the largest value (bottom) in $2 r / \eta$ which are listed in Table 1 where $2 r$ corresponds to $\ell_{n}$. Solid lines represent the curves given by the present theory with $\mu=0.345\left((1-q) \ln \delta=0.299, \alpha_{0}=1.20\right.$, $X=0.411)$. Note that $q=-0.728$.
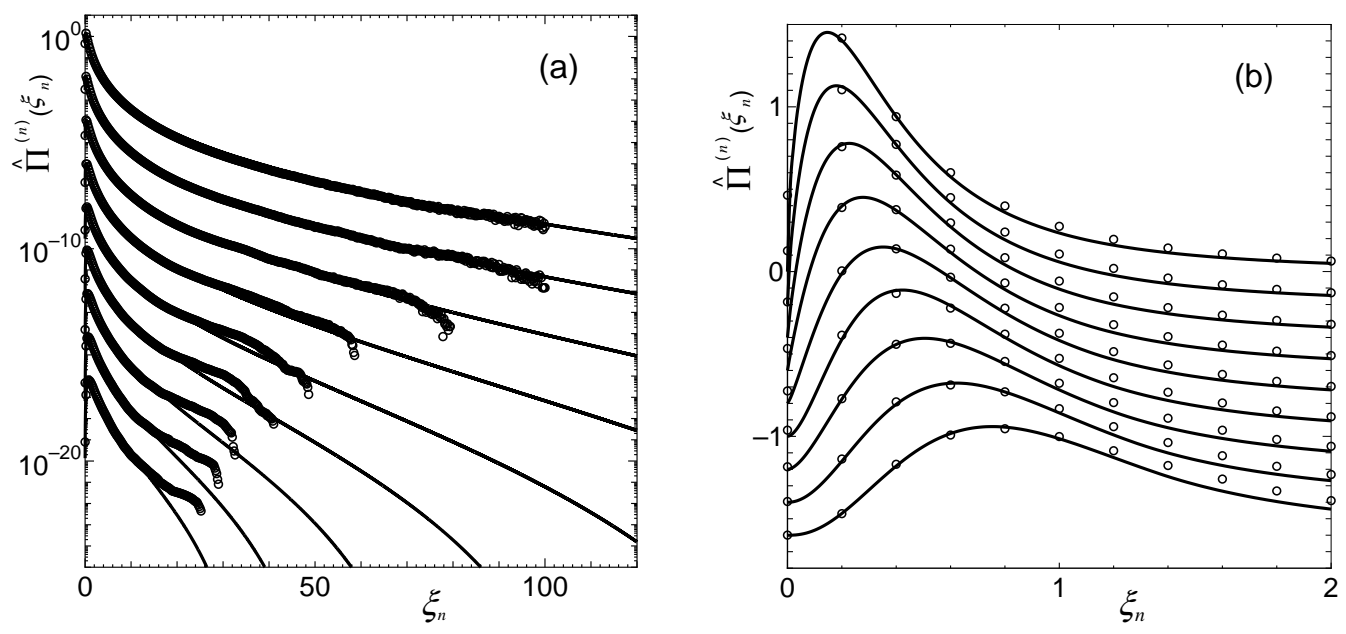

Figure 2: The PDFs of energy dissipation rates for $\delta=2^{1 / 2}$ on (a) $\log$ and (b) linear scale in the vertical axes. For better visibility, each PDF is shifted by -2 unit along the vertical axis in (a) and by -0.2 unit along the vertical axis in (b). Open circles are the DNS data points for $2 r / \eta$ corresponding to the series A for the case $\delta=2^{1 / 2}$ in Table 1 where $2 r$ corresponds to $\ell_{n}$. Solid lines represent the curves given by the present theory with $\mu=0.345\left((1-q) \ln \delta=0.299, \alpha_{0}=1.20, X=0.411\right)$. Note that $q=0.136$. 

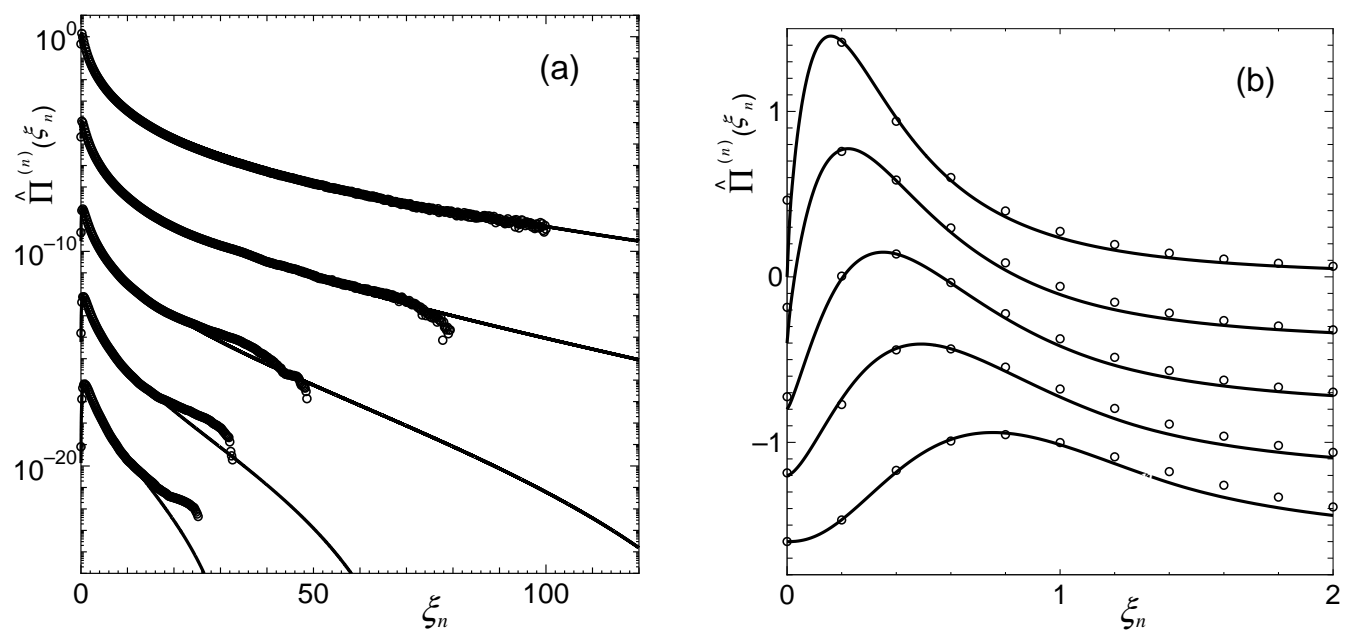

Figure 3: The PDFs of energy dissipation rates for $\delta=2$ on (a) log and (b) linear scale in the vertical axes. For better visibility, each PDF is shifted by -4 unit along the vertical axis in (a) and by -0.4 unit along the vertical axis in (b). Open circles are the DNS data points for $2 r / \eta$ corresponding to the series A for the case $\delta=2$ in Table 1 where $2 r$ corresponds to $\ell_{n}$. Solid lines represent the curves given by the present theory with $\mu=0.345\left((1-q) \ln \delta=0.299, \alpha_{0}=1.20, X=0.411\right)$. Note that $q=0.568$.

present turbulent system. The original $q$ is interpreted as a "bare" entropy index which takes the values $q=-0.728$ for $\delta=2^{1 / 4}(=1.19), q=0.136$ for $\delta=2^{1 / 2}(=1.41)$ and $q=0.568$ for $\delta=2$. Other parameters extracted by the analyses of the PDFs from DNS with the theoretical PDFs are listed in Table 1 for each $\delta$. There are two distinct series of observations $\mathrm{A}$ and B for the magnification $\delta=2^{1 / 2}$, whereas there are four distinct series A, B, C and D for $\delta=2$.

The dependences of the values of $\tilde{n}$ on $2 r / \eta=\ell_{n} / \eta$, listed in Table 1 , are shown in Fig. 4 . The formulae for the dependences are extracted by the method of least squares for $\delta=2^{1 / 4}, \delta=2^{1 / 2}$ and $\delta=2$ as

$$
\begin{aligned}
& \tilde{n}=-0.997 \ln \left(\frac{2 r}{\eta}\right)+8.68 \\
& \tilde{n}=-0.997 \ln \left(\frac{2 r}{\eta}\right)+8.68 \\
& \tilde{n}=-1.04 \ln \left(\frac{2 r}{\eta}\right)+8.86,
\end{aligned}
$$

respectively. Here, the Kolmogorov scale $\eta$ for the DNS is $\eta=5.12 \times 10^{-4}$ [30]. Since these formulae are almost common for every $\delta$, we draw in Fig. 4 the line

$$
\tilde{n}=-1.01 \ln \left(\frac{2 r}{\eta}\right)+8.74
$$

for reference. This independence of $\delta$ proves the correctness of the assumption that $\tilde{n}$ is independent of $\delta$. From Fig. 4, we find the remarkable outcome that there exists a scaling behavior extended to smaller region from the inertial range that is the range between the vertical dash-dotted lines, i.e., $126<2 r / \eta<448$ [30]. This may be attributed to the fact that MPDFT is the theory looking at the singularities which become more conspicuous as $2 r\left(=\ell_{n}\right)$ getting smaller (see the discussion in section 2 ).

The comparison of the extracted formula (96) for $\tilde{n}$ with the theoretical relation

$$
\tilde{n}=-\ln \left(\frac{2 r}{\eta}\right)+\ln \left(\frac{\ell_{0}}{\eta}\right)
$$




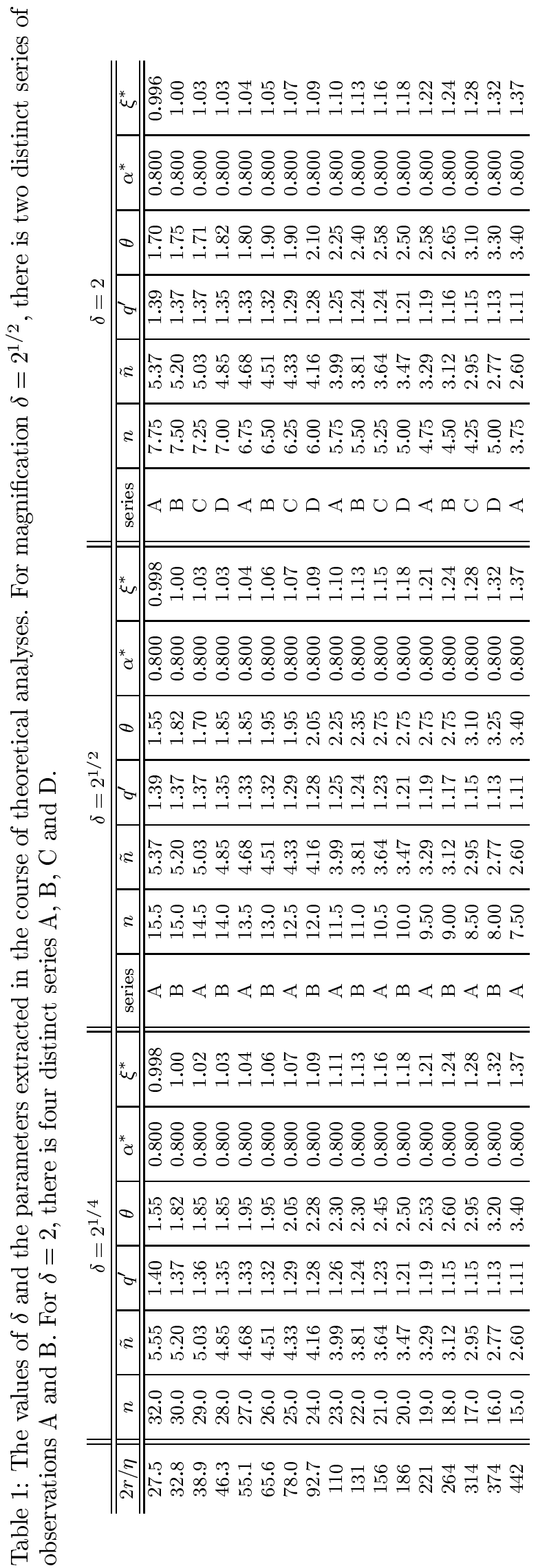




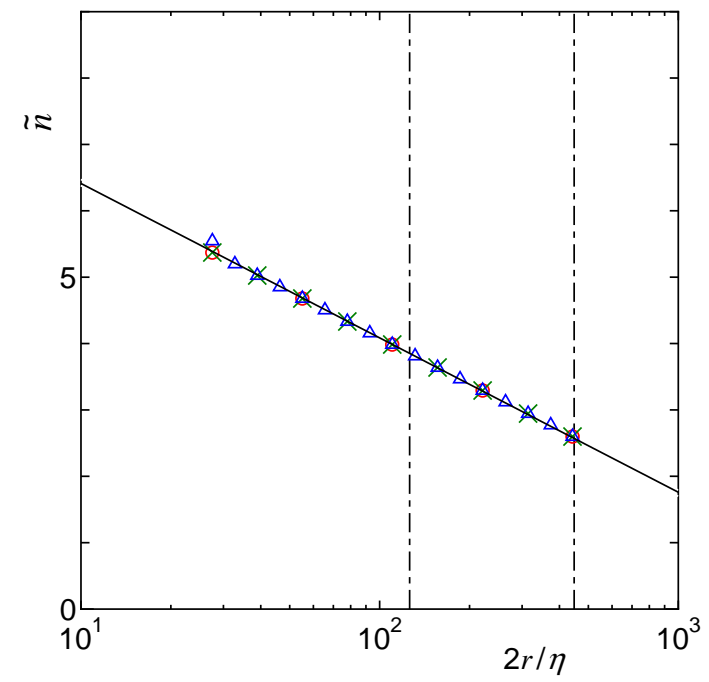

Figure 4: The relation between $\tilde{n}$ and $2 r / \eta$ extracted from the PDFs of energy dissipation rates for $\delta=2^{1 / 4}$ (open triangles), for $\delta=2^{1 / 2}$ (crosses; the series A in Table 1) and for $\delta=2$ (open circles; the series $\mathrm{A}$ in Table 1$)$. The line is $\tilde{n}=-1.01 \ln (2 r / \eta)+8.74$. Here, $2 r$ corresponds to $\ell_{n}$. Note that the inertial range is the region between the vertical dash-dotted lines.

provides us with the estimation $\ell_{0} / \eta=3.20 \times 10^{4}$. Since the smallest grid spacing is $3 \eta[30], \ell_{0} / 3 \eta=$ $1.07 \times 10^{4}$ provides us with the number of grids corresponding to $\ell_{0}$. Note that the estimated value of $\ell_{0} / 3 \eta$ is about 5.2 times larger than the possible largest meaningful length 2048 in the unit of the number of grids due to the periodic boundary condition of the DNS. Note also that $\ell_{0} / \eta$ is about 8 times larger than the integral length $L / \eta=2.130 \times 10^{3}$ of the DNS under consideration [30].

The values $\alpha^{*}$ of the connection point are constant with respect to $2 r / \eta$ and to $\delta$ (see Table 1 ). The corresponding values for the connection point $\xi_{n}^{*}$ are given by (78) with (72) and (89) for $\phi=3$, and are listed in Table 1. The dependence of $\xi_{n}^{*}$ on $2 r / \eta$ is extracted out by the method of least squares with the help of whole the data from $\delta=2^{1 / 4}, \delta=2^{1 / 2}$ and $\delta=2$ in the forms

$$
\xi_{n}^{*}=4.42 \times 10^{-2}\left[\ln \left(\frac{2 r}{\eta}\right)\right]^{2}-0.293 \ln \left(\frac{2 r}{\eta}\right)+1.49,
$$

respectively. This line is drawn in Fig. 5. It is remarkable that the specific dependence of the connection point $\xi_{n}^{*}$ on $2 r / \eta$ is found for wider region, extended to smaller values with respect to $2 r / \eta$, than the inertial range which is the region between the vertical dash-dotted lines [30]. Note that the extracted formula has rather simple form in spite of the fact that $\xi_{n}^{*}$ has a complicated dependence on $2 r / \eta$ due to the implicit dependences through the quantities (72) and (89) in addition to the explicit dependence in (78).

The dependences of the entropy index $q^{\prime}$ of the PDFs $(\phi=3)$ for the central part (83) with (92) on $2 r / \eta=\ell_{n} / \eta$, listed in Table 1 , are shown in Fig. 6 . The formulae for the dependences are extracted by the method of least squares for $\delta=2^{1 / 4}, \delta=2^{1 / 2}$ and $\delta=2$ as

$$
\begin{aligned}
& q^{\prime}=-0.100 \ln \left(\frac{2 r}{\eta}\right)+1.73 \\
& q^{\prime}=-0.102 \ln \left(\frac{2 r}{\eta}\right)+1.73 \\
& q^{\prime}=-0.0934 \ln \left(\frac{2 r}{\eta}\right)+1.69
\end{aligned}
$$




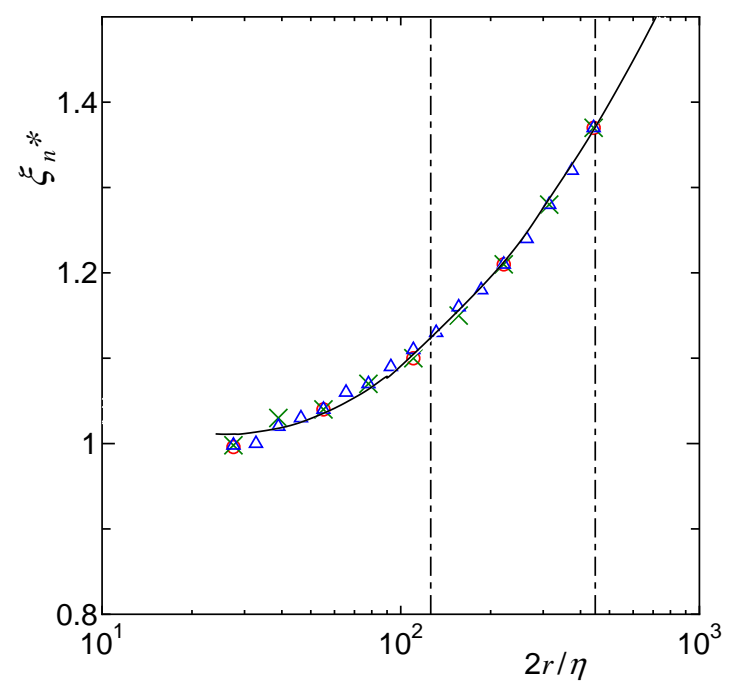

Figure 5: The relation between $\xi_{n}^{*}$ and $2 r / \eta$ extracted from the PDFs of energy dissipation rates for $\delta=2^{1 / 4}$ (open triangles), for $\delta=2^{1 / 2}$ (crosses; the series A in Table 1) and for $\delta=2$ (open circles; the series A in Table 1). The line is $\xi_{n}^{*}=4.42 \times 10^{-2}[\ln (2 r / \eta)]^{2}-0.293 \ln (2 r / \eta)+1.49$. Here, $2 r$ correspond to $\ell_{n}$. Note that the inertial range is the region between the vertical dash-dotted lines.

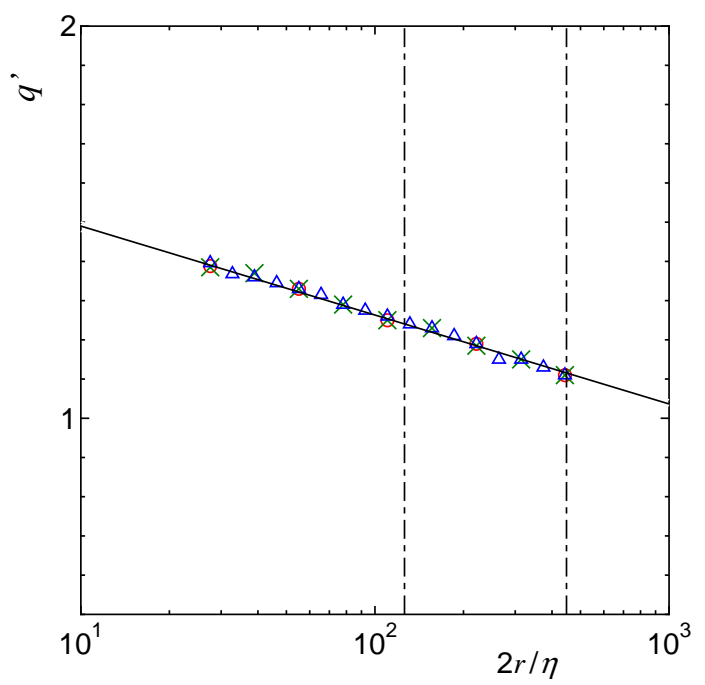

Figure 6: The relation between $q^{\prime}$ and $2 r / \eta$ extracted from the PDFs of energy dissipation rates for $\delta=2^{1 / 4}$ (open triangles), for $\delta=2^{1 / 2}$ (crosses) and for $\delta=2$ (open circles). The line is $q^{\prime}=-0.0985 \ln (2 r / \eta)+1.72$. Note that the inertial range is the region between the vertical dash-dotted lines. 


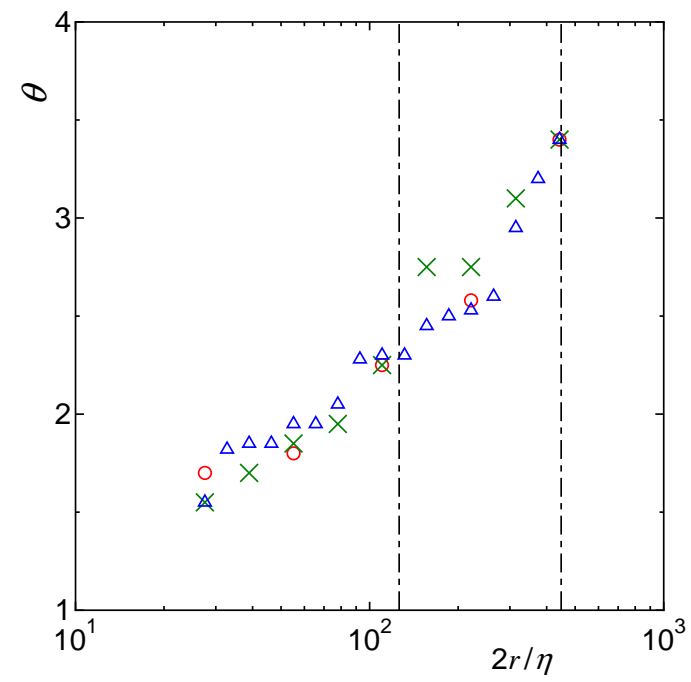

Figure 7: The relation between $\theta$ and $2 r / \eta$ extracted from the PDFs of energy dissipation rates for $\delta=2^{1 / 4}$ (open triangles), for $\delta=2^{1 / 2}$ (crosses) and for $\delta=2$ (open circles). Note that the inertial range is the region between the vertical dash-dotted lines.

respectively. Since these formulae are almost common for every $\delta$, we draw in Fig. 6 the line

$$
q^{\prime}=-0.0985 \ln \left(\frac{2 r}{\eta}\right)+1.72
$$

for reference.

As can be seen in Fig. 7, the values of $\theta$ are rather scattered for the present analyses of the energy dissipation rates, which may be attributed to the lack of data points for the experimental PDFs near $\xi_{n}=0$. We expect that, with the analyses near the center part of PDFs based on more data points, one can extract the more reasonable characteristics of fluctuations around the coherent turbulent motion. The results of the reexamination in this direction will be published elsewhere in the near future.

\section{Summary and prospects}

The PDFs for energy dissipation rates, extracted out from $4096^{3}$ DNS [30], are analyzed in terms of MPDFT in a high accuracy for several values of $\delta$ (see Fig 1, Fig. 2 and Fig. 3 ). The PDFs from the DNS had been provided as the series with the magnification $\delta=2^{1 / 4}$. From the original series, we created two series with magnifications $\delta=2^{1 / 2}$ and $\delta=2$ in order to check if the choice of $\delta$ does not affect observables of the turbulent system within the analysis by MPDFT with the new scaling relation. Here, observables are the multifractal spectrum, the mass exponents, the scaling exponents and so on. As the value $\delta(>1)$ is chosen arbitrarily by observers when they produce a series of PDFs, observables of a turbulent system should not depend on $\delta$. With the new scaling relation, the independence of observables on $\delta$ has been proven through the accurate analyses of PDFs performed in this paper. It is also revealed that $\tilde{n}$ does not depend on $\delta$, which ensures the uniqueness of the PDF of $\alpha$ for any value of $\delta$.

It is found from the dependence of $\tilde{n}$ on $2 r\left(=\ell_{n}\right)$ that the scaling behavior extends itself to the region where $2 r$ is smaller than the inertial range (see Fig. 4). It is reasonable in the sense that MPDFT is the formalism analyzing the singularities of those observables responsible for intermittency which is important for smaller $\ell_{n}$. The dependence of the connection point $\xi_{n}^{*}$ on $2 r$ shows a specific behavior extended to smaller region than the inertial range (see Fig. 5). The information of the connection point $\xi_{n}^{*}$ may be useful for wavelet analysis to separate the coherent motion of eddies in turbulence and the fluctuation around eddies. 
It is revealed that $\alpha^{*}$ at the connection point are constant with respect to $2 r$ and $\delta$. In this paper, we adjusted the connection point $\alpha^{*}$, therefore $\xi_{n}^{*}$, in order for the best fit of the PDF around the region between the peak and the connection point. In the previous analyses, we had chosen the connection point under the criterion that the $\operatorname{PDF} \hat{\Pi}_{\phi}^{(n)}\left(\xi_{n}^{*}\right)$ at the connection point should have the least dependence on $n$. The values $\alpha_{\text {old }}^{*}$ thus determined are also constant with respect to $2 r$, but are different from the values $\alpha^{*}$ extracted through the adjustment performed in the present paper. For the case of the PDFs for energy dissipation rates with $\delta=2$ investigated in this paper, $\alpha^{*}=0.800$ (see Table 1 ) and $\alpha_{\text {old }}^{*}=0.922$. Note that the previous analyses had been done only for $\delta=2$.

It is found that $q^{\prime}$ has the scaling behavior with respect to $2 r / \eta$, which extends to the smaller region than the inertial range (see Fig. 6). There is no theoretical prediction yet for the fluctuations around the coherent turbulent motion providing intermittency. The discovery of the scaling behavior of $q^{\prime}$ may provides us with a powerful guide to extract the characteristics of the fluctuations.

We see that the new scaling relation proposed in this paper is deeply related to the one extracted in a dynamical system, say the system of logistic map. Then, we observe that the origin of the multifractal character of the fully developed turbulence is deeply related to the $\delta$-scale Cantor sets created from $\delta^{\infty}$ periodic orbits. From the present analyses, we conjecture that the system of the fully developed turbulence consists of the accumulation of the Cantor sets characterized by unstable $\delta^{\infty}$ periodic orbits with different values of $\delta$. Observation of the system with the magnification $\delta$ extracts the information of the $\delta$-scale Cantor sets constituting the turbulence. Further investigations to this direction is now in progress, and will be given elsewhere in the near future.

\section{Acknowledgements}

The authors are grateful to Dr. Y. Kaneda and Dr. T. Ishihara for their kindness to provide them with the PDF data for the energy dissipation rates extracted out from their $4096^{3}$ DNS of fully developed turbulence. They also thank to Dr. T. Motoike, Dr. K. Yoshida, Mr. M. Komatsuzaki and Mr. K. Takechi for fruitful discussions.

\section{References}

[1] B.B. Mandelbrot, J. Fluid Mech. 62 (1974) 331.

[2] U. Frisch and G. Parisi, Turbulence and predictability in geophysical fluid dynamics and climate dynamics, North-Holland, New York, 1985, p. 84.

[3] R. Benzi, G. Paladin, G. Parisi and A. Vulpiani, J. Phys. A: Math. Gen. 17 (1984) 3521.

[4] T.C. Halsey, M.H. Jensen, L.P. Kadanoff, I. Procaccia and B.I. Shraiman, Phys. Rev. A 33 (1986) 1141.

[5] C. Meneveau and K. R. Sreenivasan, Nucl. Phys. B (Proc. Suppl.) 2 (1987) 49.

[6] M. Nelkin, Phys. Rev. A 42 (1990) 7226.

[7] I. Hosokawa, Phys. Rev. Lett. 66 (1991) 1054.

[8] R. Benzi, L. Biferale, G. Paladin, A. Vulpiani and M. Vergassola, Phys. Rev. Lett. 67 (1991) 2299.

[9] Z-S. She and E. Leveque, Phys. Rev. Lett. 72 (1994) 336.

[10] T. Arimitsu and N. Arimitsu, Phys. Rev. E 61 (2000) 3237.

[11] T. Arimitsu and N. Arimitsu, J. Phys. A: Math. Gen. 33 (2000) L235. [CorRigendum: 34 (2001) 673.]

[12] N. Arimitsu and T. Arimitsu, J. Korean Phys. Soc. 40 (2002) 1032. 
[13] T. Arimitsu and N. Arimitsu, Physica A 305 (2002) 218.

[14] L. Biferale, G. Boffetta, A. Celani, B.J. Devenish, A. Lanotte, and F. Toschi, Phys. Rev. Lett. 93 (2004) 064502.

[15] T. Arimitsu and N. Arimitsu, AIP Conf. Proc. 695 (2003) 135.

[16] T. Arimitsu and N. Arimitsu, Physica D 193 (2004) 218.

[17] T. Gotoh and R.H. Kraichnan, Physica D 193 (2004) 231.

[18] T. Arimitsu and N. Arimitsu, Physica A 340 (2004) 347.

[19] T. Arimitsu and N. Arimitsu, Journal of Physics: Conference Series 7 (2005) 101.

[20] L. Chevillard, B. Castaing, E. Lévêque and A. Arneodo, Physica D 218 (2006) 77.

[21] T. Arimitsu, N. Arimitsu, K. Yoshida and H. Mouri, Anomalous Fluctuation Phenomena in Complex Systems: Plasma Physics, Bio-Science and Econophysics, Research Signpost, India, 2008, p. 25; and the references therein.

[22] A.M. Oboukhov, J. Fluid Mech. 13 (1962) 77.

[23] A.N. Kolmogorov, J. Fluid Mech. 13 (1962) 82.

[24] A.M. Yaglom, Sov. Phys. Dokl. 11 (1966) 26.

[25] U.M.S. Costa, M.L. Lyra, A.R. Plastino and C. Tsallis, Phys. Rev. E 56 (1997) 245.

[26] M.L. Lyra and C. Tsallis, Phys. Rev. Lett. 80 (1998) 53.

[27] A. Rényi, Proceedings of the 4th Berkeley Symposium on Mathematical Statistics and Probability, 1961, p. 547.

[28] J.H. Havrda and F. Charvat, Kybernatica 3 (1967) 30.

[29] C. Tsallis, J. Stat. Phys. 52 (1988) 479.

[30] T. Aoyama, T. Ishihara, Y. Kaneda, M. Yokokawa, K. Itakura and A. Uno, J. Phys. Soc. Jpn. 74 (2005) 3202.

[31] A.N. Kolmogorov, Dokl. Akad. Nauk. SSSR 30 (1941) 301.

[32] A.N. Kolmogorov, Dokl. Akad. Nauk. SSSR 31 (1941) 538.

[33] G.B. Bagci and U. Tirnakli, Phys. Lett. A 373 (2009) 3230.

[34] A.S. Parvan and T.S. Biro, Phys. Lett. A 374 (2010) 1951. 